\title{
A Case Report of Transient Exertional Hypoxemia in Pregnancy and Review of Hereditary Hemorrhagic Telangiectasia
}

\author{
Matthew Janssen ${ }^{1 *}$, Kimia Menhaji ${ }^{1}$, Dominic Marchiano ${ }^{1}$ and Lauren Catalano ${ }^{2}$ \\ ${ }^{1}$ Department of Obstetrics and Gynecology, Pennsylvania Hospital, Philadelphia, USA \\ ${ }^{2}$ Department of Pulmonology, Pennsylvania Hospital, Philadelphia, USA
}

${ }^{*}$ Corresponding author: Matthew Janssen, Obstetrician/Gynecologist, Department of Obstetrics and Gynecology, Pennsylvania Hospital 800 Spruce Street, 2 Pine East Philadelphia, PA 19107, USA, Tel: +001 267-584-1843; E-mail: Matthew.janssen@uphs.upenn.edu

Rec date: November 13, 2018; Acc date: November 28, 2018; Pub date: November 30, 2018

Citation: Janssen M, Menhaji K, Marchiano D, Catalano L (2018) A Case Report of Transient Exertional Hypoxemia in Pregnancy and Review of Hereditary Hemorrhagic Telangiectasia. Gynecol Obstet Case Rep Vol.4 No.3:68.

\section{Abstract}

A 27-year-old G3P1011 presented at 34 weeks gestation with dyspnoea, which she also reported in her prior pregnancy. Her dyspnoea was accompanied by exertional hypoxemia. After excluding other etiologies of hypoxia such as cardiac anomalies and pulmonary embolism, arterio-venous shunting became the working diagnosis. She was treated successfully with ambulatory oxygen therapy and her symptoms resolved spontaneously after delivery with a good maternal and fetal outcome. Although we were unable to confirm a diagnosis of arteriovenous malformation of hereditary hemorrhagic telangiectasia, we remain suspicious that this is the underlying cause of her symptoms. Review of hereditary hemorrhagic telangiectasia identifies concerns regarding screening and obstetric management. Hereditary hemorrhagic telangiectasia is a rare genetic disease that may present as transient exertional hypoxemia in the third trimester of pregnancy. Pregnancy with arteriovenous shunting can be managed with symptomatic treatment along and careful attention to existing obstetric and anesthetic guidelines.

Keywords: Cervical cancer; Carcinoma; Breast cancer; Menopause; Endometrial cavity; Neoadjuvant chemotherapy

Abbreviations: AVM: Arterio-Venous Malformation; HHT: Hereditary Hemorrhagic Telangiectasia; JVD: Jugular Venous Distention; PVOD: Peripheral Vaso-Occlusive Disease

\section{Introduction}

It is expected that approximately $76 \%$ of women report shortness of breath by the third trimester and that the vast majority of them are benign gestational dyspnoea [1]. However, it is important to remember that other serious cardiopulmonary causes can manifest with the same presenting complaint. In our case we identified a patient with recurrent transient exertional hypoxia isolated in pregnancy. This is a rare finding that presents a puzzling clinical picture. Cardiopulmonary causes are the most common explanation for this finding and include cardiac failure and pulmonary embolus. These etiologies result in right-left shunting that creates ventilation-perfusion mismatch and subsequent hypoxemia. Another important differential diagnosis is pulmonary arterio-venous malformations, which may be the result of a genetic disorder known as hereditary hemorrhagic telangiectasia (HHT). This is a rare genetic disease yet over $50 \%$ of isolated pulmonary arterio-venous malformations (AVM) are associated with this condition [2]. Pregnancy with arterio-venous shunting is considered high risk but can be managed with symptomatic treatment along with careful attention to obstetric and anesthetic guidelines to facilitate good maternal and fetal outcomes. These guidelines may also be appropriate for cases of isolated AVMs outside of the constellation of HHT. Although some aspects of this rare disease are well known there is a significant lack of knowledge regarding the appropriate mode of delivery as well as intrapartum anesthetic choice.

\section{Case Report}

A 27-year-old G3P1011 with past medical history significant for childhood asthma presented at 34 weeks gestation with dyspnoea on exertion. Her obstetric history includes a primary cesarean section for a herpes simplex outbreak. Starting at 29 weeks gestation she experienced orthopnea and dyspnoea with activities of daily living. Ambulatory pulse-oximetry noted desaturation to $83 \%$ on room air while walking approximately 20 feet along with immediate resolution to $100 \%$ with rest on room air. Ambulatory saturation on $2 \mathrm{~L} \mathrm{O}_{2}$ per minutemaintained saturations at $98-100 \%$. Heart and lung exam did not demonstrate elevated JVD, murmurs, pathologic heart sounds, rales, rhonchi, or wheezes. There was no evidence of clubbing, cyanosis or significant skin or joint findings. The remainder of the physical exam was within normal limits including her blood pressure, heart rate, and respiratory rate. Fetal evaluation was normal with a reactive non-stress test and estimated fetal weight at the 24 percentiles. She had a normal chest X-ray, a CT scan with normal lung parenchyma 
without evidence of pulmonary thromboembolism, and an EKG in normal sinus rhythm without axis deviation or STsegment elevation. Laboratory studies ruled out acute coronary syndrome as well as atypical preeclampsia. An echocardiogram showed mild tricuspid and pulmonary valvular regurgitation without effusion and a left-ventricular ejection fraction of $60-65 \%$ with a normal right ventricular systolic pressure of $18.8 \mathrm{mmHg}$. She was admitted to the antepartum service for further workup of her hypoxemia.

Interestingly, she reported that these symptoms mimicked those in her previous pregnancy six years prior. She reported having a cardiology workup with a normal echocardiogram with ejection fraction of $60 \%$, electrocardiogram, and Holter monitor during that pregnancy without abnormal findings. She saw a cardiologist postpartum, with complete resolution of her symptoms and without a diagnosis.

During the current pregnancy, she presented to the $\mathrm{OB}$ triage unit at 9 weeks gestation with complaints of chest pain. She had normal vitals and oxygen saturation. A cardiac workup including an EKG, troponin, TSH and CXR were within normal limits. Additionally, she presented to triage at 15 weeks gestation with a presumed viral upper respiratory infection. At that time, she was documented to have no $\mathrm{O}_{2}$ requirement at rest or ambulation and discharged with an oseltamivir prescription.

Pulmonology was consulted and recommended evaluating for arterio-venous shunt. This was performed with an ECHO bubble study, which was negative for inter-atrial shunting. Rheumatologic labs including ANA, ANCA, and rheumatoid factor were negative. The patient was stable on ambulatory oxygen and discharged with home oxygen, antenatal testing, and pulmonology follow-up. Differential at this time included pulmonary embolism (PE), isolated pulmonary AVM, HHT, or peripheral vaso-occlusive disease (PVOD). Further definitive studies such as a contrast shunt CT were delayed until the postpartum period. She was recommended for repeat cesarean section given potential for worsening cardiopulmonary status with labor exertion. She continued on $2 \mathrm{~L}$ per minute ambulatory oxygen until delivery at 37 weeks 2 days by repeat cesarean section after presenting with decreased fetal movement. Placental pathology revealed no abnormal vascularity and no evidence of chronic hypoxia or infarction.

By post-operative day number 2 she was stable on room air with ambulatory saturations of $98 \%$. She was scheduled for further pulmonary function tests postpartum but has been lost to follow up despite multiple attempts to contact. It is important to realize the limitations that this loss to follow up had on the ability to cement a diagnosis and unify the constellation of findings that we present.

\section{Discussion}

Many women report dyspnoea during pregnancy. Welldescribed normal alterations in respiratory physiology with increased tidal volume and decreased functional residual capacity accounts for the majority of these symptoms [1].
Occasionally these symptoms are harbingers for other conditions. Given the combination of dyspnoea on exertion with transient exertional hypoxemia, our patient warranted further investigation. Our initial differential diagnosis included $P E$, cyanotic cardiac malformation, pulmonary hypertension, arterio-venous malformation, or PVOD.

Accordingly, we eliminated diagnoses based on results of further clinical tests. Physical exam without evidence of peripheral cyanosis, dermatologic, or rheumatologic findings eliminated PVOD. A negative CT-PE combined with a normal ECHO made structural cardiac disease, pulmonary embolism, and pulmonary hypertension significantly less likely. More invasive testing with an ECHO bubble study continued to rule out cardiac causes of shunt. At this point she was stable on a relatively benign intervention of $\mathrm{O}_{2}$ with ambulation. Given the other risks of further radiation and contrast to investigate for pulmonary and extra-thoracic shunts we decided to continue to treat her symptoms with $\mathrm{O}_{2}$ and her pregnancy and delivery as if she possessed either an isolated AVM or HHT.

We came to this conclusion because on review of the literature the most common disorder associated with exertional hypoxia is HHT. Also known as Osler-Weber- Rendu syndrome, this autosomal dominant disease affects 1 in 5,000 people [3]. The mutations in patients with HHT produce abnormal TGF- $\beta$ proteins that cause an inability to properly recruit smooth muscle cells during angiogenesis leading to AVMs [4]. A common diagnostic criterion used to identify patients with HHT is the Curacao criteria. This involves four specific characteristics such as: frequent epistaxis, first-degree relative with $\mathrm{HHT}$, mucocutaneous telangiectasia, and visceral AVMs. A diagnosis is definite if three criteria are present, suspected with two, and unlikely if less than two criteria are met [5].

Our patient fails the Curacao criteria given that none of these criteria are filled. The Curacao criteria are also well validated with a negative predictive value of $97.7 \%$ in one study of 465 patients with a gold standard of diagnostic genetic testing [6]. We note that the workup for our patient at this time is incomplete and requires further studies such as pulmonary angiography, imaging for extrathoracic AVMs (hepatic, cerebral, spinal), and pulmonary function testing. It should be noted that the CT performed was not designed to discover pulmonary arterio-venous malformations but instead for pulmonary embolism. The specific radiologic protocol for this analysis was purposefully delayed to limit contrast and radiation exposure during pregnancy as the utility of finding a specific AVM seemed fruitless as symptoms were well controlled on ambulatory oxygen. However, she has unfortunately been lost to follow-up at this point, despite multiple attempts at contact.

Although HHT is a possible diagnosis for our patient there are no findings of pulmonary AVMs on CT imaging. However, there are multiple cases of HHT with extrathoracic manifestations (notably hepatic) that could also cause dyspnoea and hypoxemia, including one rare case of vaginal, cervical, and uterine AVMs [7]. We implemented ambulatory oxygen therapy to keep $\mathrm{O}_{2}$ saturations above $95 \%$ to prevent 
fetal hypoxia as well as for symptomatic treatment. She recovered quickly postpartum with return to normal oxygen saturations by the second postoperative day. Physiologically this is supported as the systemic vascular resistance falls and blood flow through the AVM decreases along with shunt fraction. In contrast to this quick recovery to pre-pregnancy function, a single case describes symptoms of dyspnoea and vascular congestion that persisted until discharge 16 days postpartum [8].

We chose to focus the remainder of the discussion on hereditary hemorrhagic telangiectasia given a dearth of highquality evidence that describe maternal outcomes. The guidelines for management of pregnant patients with known or suspected HHT are largely derived from expert opinion and small case studies. Screening recommendations seek to identify all locations of AVMs to provide safe obstetric analgesia and delivery. The majority of patients with known AVM's were delivered by cesarean section although a number of cases demonstrate the safety of vaginal delivery. Ultimately the best route of delivery for maternal HHT is not known.

Recommendations are for patients with $\mathrm{HHT}$ to undergo screening for AVMs. The overall prevalence in the disease population in different organ system circulations is: hepatic (30\%), pulmonary (48\%), cerebral (10\%), and spinal (1\%) [7]. This screening recommendation becomes important given that a study of 111 women with HHT showed that $74 \%$ were unaware of the diagnosis prior to pregnancy [3]. Other acquired causes of AVMs exist, such as trauma, hepatic cirrhosis, schistosomiasis, and lung cancer. None of these are known to exist in our case.

Pulmonary AVMs are most likely to cause exertional hypoxia, as their presence in the low-pressure pulmonary system allows for right to left shunting (although the hepatic circulation has similar low-pressure physiology). Multiple articles describe pulmonary AVMs enlarging in pregnancy, possibly from estrogen/progesterone effects on the vasculature $[2,9]$. Combined with increased cardiac output, blood volume, and decreased systemic vascular resistance of pregnancy, an enlarged AVM may result in worsening exertional hypoxemia. A single article postulates that increasing blood volume of pregnancy, which peaks at around 30 weeks, results in selective distention of the AVM vs. nearby capillaries due to decreased resistance within the AVM. Increased relative blood flow through the AVM then increases the overall shunt fraction and causes symptoms [9]. These changes in maternal physiology also dictate the most likely time of presentation in the third trimester, as in our patient. On review of most case reports/series of HHT in pregnancy, the presenting symptom is most often related to dyspnoea or rupture of the AVM causing acute hemorrhage [7]. Another case presented with "worsening hypoxemia" with "limited exercise tolerance" [5].

The decision to proceed with cesarean delivery stemmed largely from concern over unknown cardiopulmonary status for our patient. This decision was supported in a systematic review by Lomax et al. which demonstrated that $53 \%(9 / 17)$ of patients with HHT (with predominantly pulmonary AVMs) were delivered by cesarean section although the indications for cesarean delivery as well as previous delivery history are unclear. Two of these patients are also noted to deliver with forceps assistance, although the indication for this choice is not specified [4].

Regardless of mode of delivery, there are significant anesthetic complications for obstetric patients with $\mathrm{HHT}$, including worsening of maternal cardiorespiratory status, the risk of spinal AVMs causing hematoma formation, or spinalcord ischemia. Multiple articles reinforce the importance of screening for spinal AVMs to ensure safe use of regional anesthesia $[3,7]$.

Since HHT is an autosomal dominant disease there is a $50 \%$ chance of inheritance in the fetus. A single study analyzed the outcomes of 560 pregnancies and noted that anomalies were irrespective of whether the infant inherited the HHT mutation [10]. In this study, HHT in a parent did not lead to adverse perinatal outcomes, regardless of mode of delivery [10].

Only a single reported case of HHT in pregnancy presented as hypoxemia and there are no descriptions of treatment with ambulatory oxygen [5]. We present a case of recurrent exertional hypoxia in consecutive pregnancies with a mixed clinical picture with features concerning for $\mathrm{HHT}$ but without a diagnosis according to either imaging or consensus criteria. We recognize that other diagnoses could explain these clinical findings, although testing did not lead to diagnosis of any more common conditions. We focused on HHT as a possible unifying diagnosis given the constellation of symptoms; yet we also realize that this may not ultimately be the correct diagnosis. The review of HHT describes some clinical implications that may affect a woman's pregnancy including genetic inheritance as well as intrapartum anesthesia. It is important to know recommendations for screening if this disease is encountered.

\section{Conclusion}

Further research on this rare condition is needed to describe how HHT changes pregnancy physiology and may combine to exacerbate common complaints in pregnancy as well as to create more robust guidelines for both obstetricians and anesthesiologists.

\section{Conflict of Interest}

The authors have no conflicts of interest to report with this research.

\section{Informed Consent}

Informed consent taken.

\section{References}

1. Milne JA, Howie AD, Pack Al (1978) Dyspnoea during normal pregnancy. Br J Obstet Gynaecol 85: 260-263. 
2. Esplin MS, Varner MW (1997) Progression of pulmonary arteriovenous malformation during pregnancy: Case report and review of the literature. Obstet Gynecol Surv 52: 248-253.

3. Shovlin CL, Sodhi V, McCarthy A, Lasjaunias P, Jackson JE, et al. (2008) Estimates of maternal risks of pregnancy for women with hereditary haemorrhagic telangiectasia (osler-weber-rendu syndrome): Suggested Approach for Obstetric Services. BJOG 115: 1108-1115.

4. Bari O, Cohen PR (2017) Hereditary hemorrhagic telangiectasia and pregnancy: Potential adverse events and pregnancy outcomes. Int J Womens Health 9: 373-378.

5. Shovlin $\mathrm{CL}$, Guttmacher $\mathrm{AE}$, Buscarini $\mathrm{E}$, Faughnan $\mathrm{ME}$, Hyland $\mathrm{RH}$, et al. (2000) Diagnostic criteria for hereditary hemorrhagic telangiectasia (Rendu-Osler-Weber syndrome). Am J Med Genet 91: 66-67.

6. Van Gent MW, Velthuis S, Post MC, Snijder RJ, Westermann CJ, et al. (2013) Hereditary hemorrhagic telangiectasia: How accurate are the clinical criteria? Am J Med Genet A 161A: 461-466.

7. Lomax S, Edgcombe $H$ (2009) Anesthetic implications for the parturient with hereditary hemorrhagic telangiectasia. Can J Anaesth 56: 374-384.

8. Berthelot E, Savale L, Guyo A, Rahmoune FC, Bouchachi A, et al. (2015) Acute high output heart failure revealing hereditary hemorrhagic telangiectasia in a pregnant woman. Presse Med. 44: 362-365.

9. Swinburne AJ, Fedullo, AJ, Gangemi R, Mijangos JA (1986) Hereditary telangiectasia and multiple pulmonary arteriovenous fistulas. Clinical deterioration during pregnancy. Chest 89: 459-460.

10. Wain K, Swanson K, Watson W, Jeavons E, Weaver A, et al. (2012) Hereditary hemorrhagic telangiectasia and risks for adverse pregnancy outcomes. Am J Med Genet A 158A: 2009-2014. 